\title{
A Hippocratic Oath for geologists?
}

\section{Ruggero Matteucci ${ }^{1,{ }^{\star}}$, Guido Gosso ${ }^{2}$, Silvia Peppoloni ${ }^{3}$, Sandra Piacente ${ }^{4}$, Janusz Wasowski ${ }^{5}$}

\author{
${ }^{1}$ Università di Roma 'La Sapienza', Dipartimento di Scienze della Terra, Rome, Italy \\ ${ }^{2}$ Università Statale di Milano, Dipartimento di Scienze della Terra 'A. Desio', Milano, Italy \\ ${ }^{3}$ Istituto Nazionale di Geofisica e Vulcanologia, Rome, Italy \\ ${ }^{4}$ Università di Modena e Reggio Emilia, Dipartimento di Scienze della Terra, Modena, Italy \\ ${ }^{5}$ Istituto di Ricerca per la Protezione Idrogeologica, Consorzio Nazionale della Ricerca (CNR), Bari, Italy
}

\author{
Article history \\ Received January 23, 2012; accepted March 14, 2012. \\ Subject classification: \\ Hippocratic Oath, Geologists, Geoethics, Social responsibility.
}

\begin{abstract}
We argue here that the introduction of an ethical code of conduct that follows the example of the Hippocratic Oath of physicians will help geologists to acquire binding awareness of their professional and social responsibilities. The ethical behavior and obligations of modern geologists involve, but are not limited to, the following issues: correct land/environment use and management; respect of truth and science; and protection of the Earth systems, on both the local and global scales, and therefore, of our well-being. We believe that for geoligists, the explicit acceptance of an ethical code will help to promote: (i) an awareness of their social role, expertise and sense of belonging to a professional community; (ii) an understanding of the expectations of citizens and society; and (iii) cultural growth, with better use of research and implementation of scientific and professional skills. All this should enhance the public recognition of the social mission of geologists, which is essential for the well-being of society. Therefore, we suggest that like in the majority of medical schools, ethical training should be a part of the university curriculum for students in geology.
\end{abstract}

\section{The Hippocratic Oath}

The 'Hippocratic Oath', through which young physicians still today express their ethical responsibilities, represents the first written manifestation of the value inherent in the moral obligations that arise from the possession of specific knowledge that has practical consequences. With Hippocrates, medicine that had until then been hieratical and theocratic, became rational.

For a modern physician, this Oath goes beyond the rules of professional conduct that are based on the deontological code, to exalt the moral obligation of the absolute availability to intervene in case of necessity, and to legitimize the expectation of those in need of medical help. The recurrent modernization of the original text by medical organizations of different countries and the relevant literature [e.g., Reiser 2003, Gruenbaum and Jotkowitz 2009] demonstrate the persistent importance attributed to the voluntary recognition by young physicians of the ethical dimension of their actions, also in relation to the expectations of society at large.

\section{Parallelism between ethical responsibilities of physicians and geologists}

The relations between physician, illness and the patient is guided splendidly in the Hippocratic Oath, to guarantee the patient as a person (Figure 1a). Until today, this has represented the basis of the medical profession. However, in recent times, this relationship has acquired more complexity, as it is linked to its social dimension. In this context, in the triangle of the Hippocratic relations, as physician - illness - patient, there needs to be added the collectivity, and more in general, society (Figure $1 \mathrm{~b}$ ). While maintaining the rights of the patient as a person, more and more it is the social dimension (and the cost/benefit ratio) of the illness that necessarily governs biomedical research, the physician's action and physician's potential to operate. The problem of so-called 'rare illnesses' represents a clear example here (with ethical obligation to treat, but very high cost/benefit ratio for society). The social dimension of medicine represents a relatively recent acquisition. In Italy, only during Napoleon's period did the 'district physician' became a reality in all provinces, which heralded the beginning of generally available medical help in the form of a public service.

The truth is that ethics have a fundamental role in the responsibilities of geologists. The Earth is our unique home: our well-being, and also our survival, depends on its habitability and resources. Thus, the Earth systems (geosphere, biosphere, hydrosphere, atmosphere) repre- 


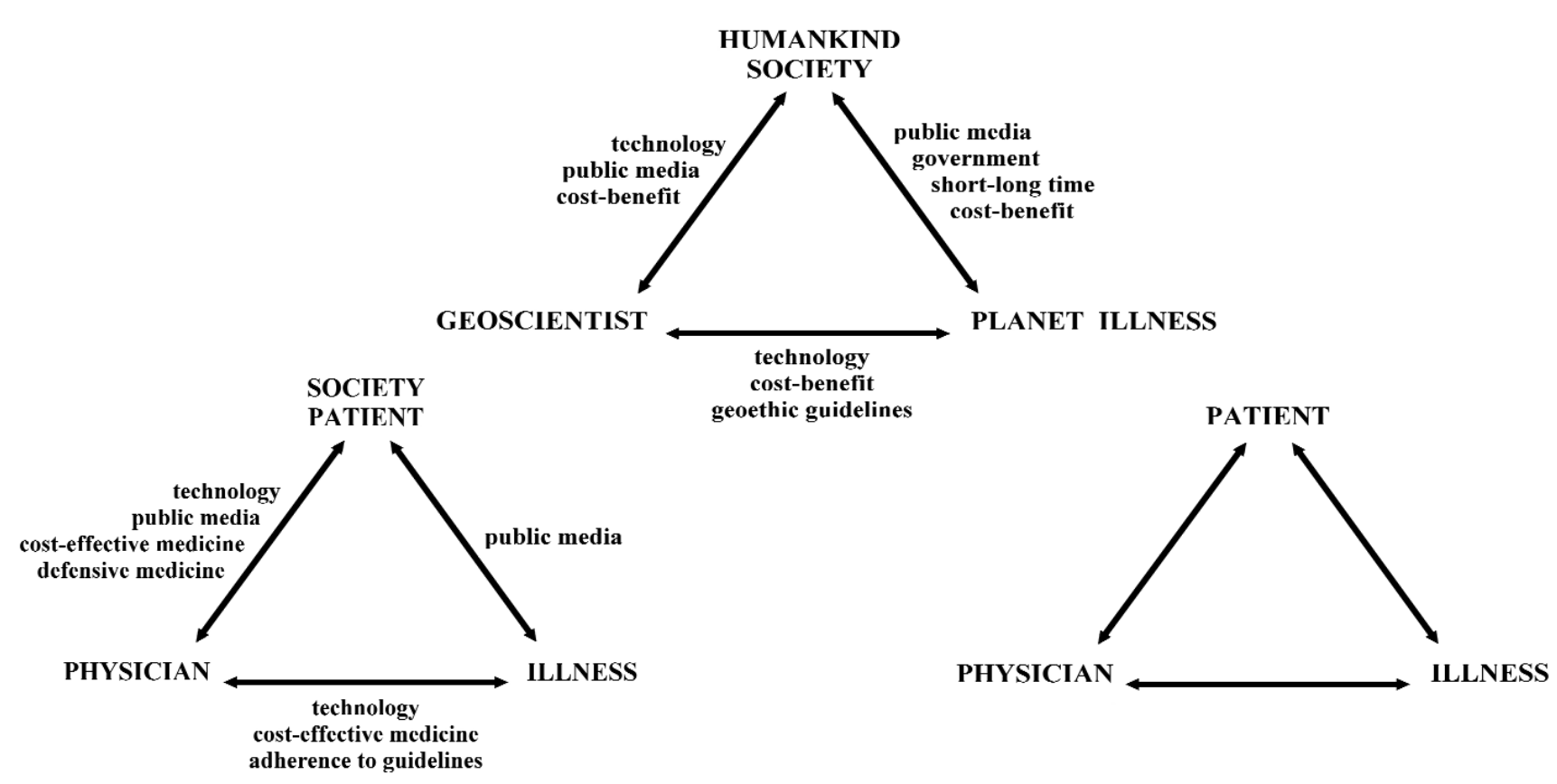

Figure 1. Factors affecting the Hippocratic triangle [inspired by Antoniou et al. 2010], in medicine (physician, illness, patient/society) and the geological sciences (geoscientists, Planet 'illness' - hazards, human imprinting - and society). Bottom right: The early relationships according to Hippocrates. Bottom left: In medicine today. Top: The geoscientists equivalent.

sent the value of the common weal. Furthermore, our Planet is not an immobile body; it has its own life in continuous evolution, with processes that are often unavoidable and damaging for man. Adequate investigation and timely transfer of information would in most cases imply lower levels of risk to man arising from such processes. It is also evident that modifications of the Earth system processes that are potentially damaging for humankind can be, and are being, induced by man, through actions that do not respect our Planet's natural processes and equilibria. The social role of the responsibility and actions of geologists is therefore extraordinarily important. Allman's activity involving land use, and more in general, the environment, should be confronted with the historical perspectives of geologists to long-term threats to our future.

Geologists have, therefore, relevant responsibilities towards society, from which, in turn, the ethical importance of their actions is derived. A correct and scientifically sound approach can mitigate, or at least help to avoid, many of the serious consequences that continuously arise through the irresponsible use of land by man. Even if geologists have limited power to impose the correct choices on decision makers, their ethical obligation is to propose them and to report wrong actions and behavior.

Therefore, the parallelism between the Hippocratic obligations of a physician with respect to society and those of geologists is evident. By analogy, the Hippocratic triangle of physician - illness - patient/society corresponds to the relations between geologists, 'Earth's illness' and society (Figure 1c), where 'Earth's illness' is represented by both natural processes that are actually or potentially damaging for man and the similarly damaging effects of man's impact on our Planet.

Unlike national and international health organizations, those of the Earth sciences do not yet have a great impact, or the ability to orient the actions of governments. It appears that only the U.S. Geological Survey (USGS), which was established in 1879 in North America, has relevant social and political influence.

The current relatively limited influence of the Earth sciences community on land management practices also relates to the inadequate and volatile perception by public opinion and politicians of the extraordinary significance in the medium-to-long term of the human and economic costs that can derive from the insufficient consideration given to geological constraints. And yet, the 172,359 victims of geo-hydrological disasters and earthquakes in Italy in the last 100 years [Prestininzi 2011] represents a tragic reality. Moreover, if on the one hand the recurrence of the so-called natural catastrophes has not resulted in significant behavioral changes in society, on the other hand, geologists have not yet been able to force public opinion and political decision makers to clearly distinguish between what is natural and inevitable (geologic events, the effects of which can be mitigated via prevention in many cases), from what is not inevitable (abuse of land and lack of respect for geological processes), and is typically due to ignorance or an overwhelming desire for short-term economic gain.

\section{A(geo)ethical approach to the profession of geologist}

The professional role of geologists is already well rooted in the framework of intellectual professions. In Italy, the practice of geology is regulated by law and through the Professional Association of Geologists, with 
its specific deontological code that was issued in 2006 and amended in 2010. The code contains a clear and explicit ethical reference to the public and the general interests of the profession (Articles 1 and 36).

Indeed, in most cases, the professional activities of geologists are linked to the general interests of the territory involved as a system, considered in the medium-tolong term rather than in the short term, and in the framework of the legislation in force. These interests might include, for example: assessment of sustainability and of long-term effects of the removal of river-bed material; evaluation of site suitability for storage of contaminated material or of radioactive waste; stabilization of a landslide through expensive structural measures or through 'natural', and probably cost-effective, drainage systems; land-use planning; and geological heritage and landscape conservation. While conducting a cost-benefit analysis of any project involving our environment, it is of prime ethical importance to evaluate if, and make sure that, the benefits are significantly greater than the costs, and not only in the short term, but also in the medium-tolong term (Figure 2). The longer temporal framework needs to be considered within the economic-patrimonial logic [Roda 2011], thus leading to the maximum benefit of man's activity at the lowest cost to the territory (economic criterion); however, such benefit has to be secured also for the future (patrimonial or hereditary criterion). Therefore, geologists have a unique obligation to guide actions aimed at the conservation of the geosphere and its 'liveableness' for future generations.

Moreover, the ethical value of the expertise of geologists has global implications, because it contributes to sustainable management of a very-high-population-density, overly consumeristic, post-industrial world, in which the disequilibrium between demand and offer is growing (e.g. regarding food, energy, water, health), fueled by irrational management and over-exploitation of resources, and by the enormous inequality in the global wealth distribution.

\subsection{Ethical obligations}

The importance of promoting ethical behavior within the geosciences community is well highlighted in the conclusions of the final report of the GSA (Geological Society of America) Presidential Conference [Horten 1997]: "Individual integrity is not enough: to be truly ethical, one must have personal integrity as well as on-going awareness and insight into the ethical problems that exist throughout the geoscience profession. In other words, geoscientists must become alert to, and active in, the subject of ethics in order for the practice of geology to be truly ethical".

There are two main fields where the ethical impact of the behavior of geologists is most dramatic:

(a) Direct actions, when a commissioned study reflects

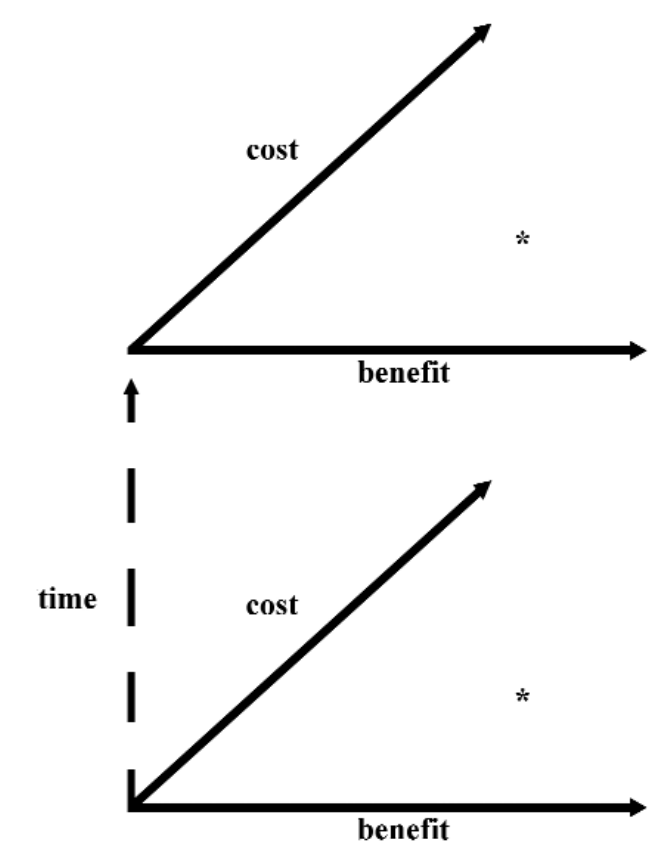

Figure 2. Long-term cost-benefit ratio in the geoethical approach.

the interests of private or public customers, but also has geoethical implications in terms of the systemic interests of the territory involved and its dynamics, and in general of our Planet (often the short-term and long-term interests of the territory and of its population do not coincide);

(b) Indirect actions, which are aimed at acquiring scientific information that will be useful for public opinion and decision makers, so that all of the actions that involve the equilibrium of the territory and our Planet and its natural evolution follow geoethical principles.

In both cases, it is also the obligation of geologists, who have a unique and adequate culture and sensitivity, to demonstrate the actual benefits (not necessarily in the short term) of the geoethically sound choice of action, which would be the most favorable for and consistent with sustainable use of the territory of interest. This might need to be confronted with the often relevant immediate benefits of less prudent choices of action. It is through this cost/benefit-based approach that it is possible to achieve respect for the core ethical values and responsible practices of geology that are consistent with the present and future well-being of society.

In particular, the Hippocratic obligations of modern geologists would involve the following:

(a) Landmanagement/use and conflicting economic interests

The ethics of sustainable land management must prevail, regardless of the type of a commissioned project and the short-term economic interests of a client. This might include, for example: exploitation of groundwater or mineral resources; engineering construction; urban development and territory management; landscape conservation; assessment of and protection from natural hazards; eval- 
uation of and protection from risks related to anthropogenic impact; conservation and enhancement of the natural and geological heritage.

\section{(b) To respect truth and science}

The respect of scientific truth and of scientific consensus based on updated evidence (including the recognition of data uncertainty, or of limited knowledge) and rigorous adherence to scientific method is a must in the professional activities of geologists. They are also ethically obliged to request a transparency policy in handling preliminary results of geological studies and in informing the public of actions that might be damaging for the territory; this obligation is even more stringent in cases of professional and scientific associations, which should therefore also adopt their own ethical code.

\section{(c) To favor the Earth, and therefore our well-being}

Although this may appear as an obvious statement, following Hippocrates, the implication is that those equipped with knowledge and specific expertise should act even outside of their professional duties to protect the Earth systems, at both the local territory and global scales.

Although the following specific 'obligations' fit into the above more general point (c), their dramatic urgency justifies their separate treatment here:

\section{(d) To promote awareness}

of every citizen's responsibility for our World

There is urgency in the need to provide adequate knowledge, so that people understand and respect the natural processes that control the life of our Planet. This is critical to orient well-informed choices that need to be made at present and in the future by individuals, society and politicians. The challenge of effective information transfer and adequate education of new generations must be won, and geologists have the ethical obligation to be at the forefront here.

(e) To assure knowledge advance and life-long learning

This critical point is also present in the Hippocratic Oath. The appreciation of the expertise of geologists by the public represents the necessary condition to promote both efficient scientific and professional action, as well as the general recognition of the role of geologists. For this reason, as for physicians, the education of geologists has to be for life. However, in addition, a high quality university education has to be assured: university studies in geology have to be recognized as one of the most complex and comprehensive educational efforts that require the integration of two areas of expertise: the natural and physical mathematical sciences. Furthermore, following the example of the majority of medical schools, ethical training should also be introduced into the university curriculum.

\subsection{The significance of an ethical obligation}

How can geologists, and especially the younger ones, be best assisted in their acquisition of a clear and binding awareness of their ethical responsibility in the geosciences? Might the introduction of an explicit act, which follows the example of the Hippocratic Oath of medical doctors, make sense here, and representan an efficient solution [Ellis and Haff 2009]? How would young geologists react if they were asked to respond on a voluntary basis to an act that they might consider as an anachronistic ritual or an imposition?

There are some strengths in the introduction and acceptance of ethical rules, as this implies a commitment to serve, using the expertise gained, for the well-being of society, which depends on land preservation and environmental protection, as well as on due respect for geophysical processes.

In the context of a publicly growing demand for ethical behavior by those who, for whatever reason, have the possibility to intervene within the public domain and to act for the public weal, the explicit acceptance of the ethical responsibility by geologists can have the following effects:

(a) Favoring explicit awareness of the social role of geologists, of their expertise and contribution, to strengthen their sense of belonging

The majority of the actions of geologists involve the public domain. In most cases important and perhaps dramatic decisions concerning geo-hazards are often more difficult to take because of possible contrasting interests and opinions; geologists have to act here as protagonists at the forefront of hazard research, analysis, and management. Regarding the questions of the global dimension (e.g. water, mineral, soil and energy resources; sea-level changes and climate variations; relations between technological solutions and respect for natural processes; cost-benefit analysis of anthropogenic intervention; assessment and mitigation of natural risks), as well as those on the regional scale (e.g. policy of geo-hydrological risk management and of protection from earthquakes and volcanic eruptions; land-use management), geologists have to have an essential role in the debate and the information transfer. We expect this will happen more often if alongside the technical-scientific dimension of the input of geologists, the public weal clearly emerges as an underlying objective. Therefore, geologists always need to act guided not only by their knowledge, but also by their awareness of the ethical implications (they need to act following 'science and conscience', exactly like physicians). Self-recognition of their role (and, consequently, recognition by others) represents 
an element of self-enhancement for geologists, and a way to increase their sense of belonging to a part of society that is essential for its survival and well-being.

(b) Fostering the awareness of geologists of the expectations of citizens and society

The significance of the Hippocratic Oath de facto entered the common feeling: citizens respect physicians and recognize their special status. Something similar is slowly starting to emerge for geologists, but their awareness of the expectations of public opinion has to go step by step along with their self-awareness of the geoethical dimension of their role.

(c) Fostering general recognition of the social mission of geologists, essential for the public weal, and, consequently, of their specific and unique role

The common feeling, at least in Italy, is that the community of geologists does not yet have levels of authority, respect and expectation that are comparable to those of some other professionals (e.g. physicians and engineers). However, the growing impact of 'planetary issues' on the media and on public opinion (e.g. unsustainable exploitation of natural resources, climate variations, pollution) and of specific regional problems linked to seismic, volcanic and geo-hydrological risks (especially in Italy, Japan, Taiwan, and in many developing countries), as well as the incipient awareness of the inadequacy of government interventions that are insufficiently supported by scientific research and by the knowledge of geophysical processes, make the conscious acceptance of responsibility by geoscientists entirely appropriate, if not necessary. Therefore, a public and solemn commitment by those who are to become experts in the management of land and of the problems of the Earth represents a useful instrument.

(d) Stimulation of the cultural growth at the individual and community levels, exploitation of research, and implementation of scientific and professional skills

The frantic acceleration in the increase of knowledge, which rapidly makes obsolete what we knew only a few years earlier, represents a demanding reality of our times. Keeping our knowledge up to date and the continuous increase in our know-how represent the necessary conditions for the practice of any profession today. However, these efforts acquire moral significance when the wellbeing of the individual and of society largely depend on the quality of the knowledge and skills of a practitioner. For geologists, excellent cultural and practical preparation has to take on an ethical dimension, starting from their university studies. Through their individual commitment, young geologists can assume the need for cultural lifelong increase as an ethical duty.

\section{Conclusions}

The explicit and conscious assumption of ethical obligations for geologists appears opportune and useful with the consideration of the urgent need to enhance the development of the geoethical dimension in the relationships between man and the geosphere. It is suggested that this can be facilitated by following the model of the Hippocratic Oath of physicians. Clearly, the transition to an operational application will need to be preceded by ample discussion and the acceptance by national and international communities of geoscientists and professionals.

\section{References}

Antoniou, S.A., G.A. Antoniou, F.A. Granderath, A. Mavroforou, A.D. Giannoukas and A.I. Antoniou (2010). Reflection of the Hippocratic Oath in modern medicine, World J. Surg., 34, 3075-3079.

Ellis, E.C., and P.K. Haff (2009). Earth Science in the Anthropocene: New Epoch, New Paradigm, New Responsibilities, Eos Trans. AGU, 9 (49), 473.

Gruenbaum, S.E., and A.B. Jotkowitz (2009). Content analysis of ethical codes written by medical students compared with other codes of medical ethics, Eur. J. Int. Med., 20, 101-104.

Horten, H. (1997). Report on: Conference on "Ethics in the geosciences", GSA Presidential Conference, Welches, Oregon; available at http: / / tierra.rediris.es / Geoethics_Planetary_Protection/GSA_Ethics_in_the_ Geosciences.pdf.

Prestininzi, A. (2011). La mancata prevenzione: costi e disagi trasferiti alle future generazioni, Geoitalia, 34, p. 3.

Reiser, S.J. (2003). What modern physicians can learn from Hippocrates, Cancer, 98, 1555-1558.

Roda. C. (2011). Geoetica, Geoitalia, 34, p. 3.

${ }^{\star}$ Corresponding author: Ruggero Matteucci,

Università di Roma 'La Sapienza', Dipartimento di Scienze della Terra, Rome, Italy; email: ruggero.matteucci@uniroma1.it.

(C) 2012 by the Istituto Nazionale di Geofisica e Vulcanologia. All rights reserved. 